\title{
Theoretical Characterization of the Hydrogen Bonding Interaction Sites of Mycolactone C Using the ONIOM Method
}

\author{
Kadjo François Kassi ${ }^{1}$, Sopi Thomas Affi ${ }^{1,3}$, N'guessan Yao Silvère Diki ${ }^{2}$, \\ Mamadou Guy-Richard Koné ${ }^{1,3, *}$, Georges Stéphane Dembélé ${ }^{1,3}$ and Nahossé Ziao ${ }^{1,3}$ \\ ${ }^{1}$ Laboratoire de Thermodynamique et de Physico-Chimie du Milieu, UFR SFA, Université Nangui Abrogoua, \\ 02 BP 801 Abidjan 02, République de Côte-d'Ivoire \\ ${ }^{2}$ Laboratoire de Constitution et Réaction de la Matière, UFR SSMT, Université Félix Houphouët-Boigny, 22 BP \\ 582 Abidjan 22, Côte d'Ivoire \\ ${ }^{3}$ Groupe Ivoirien de Recherches en Modélisation des Maladies (GIR2M)
}

\begin{abstract}
In this work, the ONIOM method, recognized for its effectiveness on large molecules, was used to determine the geometric, energetic, and spectroscopic parameters of hydrogen bond interactions of mycolactone C. Mycolactone C; one of the most virulent forms of toxin, found in Africa and Australia. It has eight (08) oxygen heteroatoms which are all hybridized $\mathrm{sp}^{2}$ and $\mathrm{sp}^{3}$. Using quantum chemistry methods, at the ONIOM level (B3LYP/6-311+G (d, p): AM1), we have determined the preferential binding sites of the hydrogen bonds in the eight mycolactone $\mathrm{C}$ oxygen heteroatoms studied. Analysis of the results revealed that the heteroatom $\mathrm{O}_{5} \mathrm{sp}^{2}$ is the most suitable site for creating a strong hydrogen bond based on the geometric, energetic (free enthalpy of complexation), and spectroscopic (vibration frequency shifts) parameters. Identifying this O5sp2 heteroatom is a significant step forward in developing a methodology for eradicating the infection and the destructive effects of this toxin.
\end{abstract}

Keywords: Mycobacterium ulcerans; Mycolactone; ONIOM; Hydrogen bond; Quantum chemistry; Buruli ulcer.

\section{Introduction}

Buruli ulcer is a disease caused by Mycobacterium ulcerans, a bacteria belonging to the family of agents responsible for tuberculosis and leprosy ${ }^{1}$. Long neglected, this disease is widely encountered in humid tropical and subtropical countries; moreover, it has experienced an upsurge in West Africa since $1980^{2}$. This situation has led the World Health Organization (WHO) to classify it as an emerging disease and recognize it as a public health and development problem ${ }^{3}$. Mycobacterium ulcerans secretes a toxin called mycolactone into skin tissue, which causes intense tissue damage due to its cytotoxic and immunosuppressive properties. Based on previous studies, six (06) different naturally occurring molecular structures of mycolactones have been isolated ${ }^{4}$. Mycolactones are the toxins produced by Mycobacterium ulcerans; they are the principal responsible agent of Buruli ulcer. Buruli ulcer is a disease spread around the world with a higher infection rate in Africa. The cytotoxic and immunosuppressive properties of mycolactones are the basis of the destruction of the skin and subcutaneous tissue. The current treatments are mainly antibiotic therapies and reconstructive surgery in cases of severe infection. The mode of action of the

*Corresponding author: Mamadou Guy-Richard Koné Email address: guyrichardkone@gmail.com DOI: http://dx.doi.org/10.13171/mjc02104261574mgrk toxin is so far unknown in medical circles. Sadly, the therapeutic arsenal against Buruli ulcer remains very limited despite the progress observed in the quality of the management and the medical care ${ }^{5}$. Antibiotic therapy and reconstructive surgery, with their high cost and numerous relapses (16 to $28 \%$ ) in cases of severe infection, remain the current standard treatments ${ }^{6}$. The lack of knowledge of the mode of action of mycolactone in the literature and the medical world hinders better management of Buruli ulcers. Most of the research work in this area is focused on the ecology of Mycobacterium ulcerans, the dermal part of the disease manifestation, synthesis, and the structural characterization of mycolactones. The relationship between the mycolactones produced by Mycobacterium ulcerans and the proteins which are indexed to be responsible for the development of Buruli ulcer is related to the conformation and the interactions between these two types of molecules. Research into the biological activities of mycolactones is essential for a good understanding of their mode of action and for establishing a new therapeutic protocol as part of preventive or even curative treatment for Buruli ulcer.

The hydrogen bond ( $\mathrm{H}$ bond) is of utmost importance for the cohesion of matter ${ }^{7}$. Indeed, it is one of the 
most important intermolecular interactions involved in supramolecular chemistry and particular proteinligand interactions ${ }^{8}$ and crystal engineering ${ }^{9,10}$. In general, the molecules are polyfunctional, as they contain several heteroatoms capable of receiving $\mathrm{H}$ bonds. Therefore, it appears important to be able to characterize the preferential site (s) of H-binding. There is, however, very little work devoted to the study of competition between different sites within a single molecular structure. Etter and Reutzel ${ }^{11}$ have been among the first research teams to classify the acceptor power of $\mathrm{H}$ binding (ALH) of different organic functionalities. More recently, some works were focused on the competition between nitrogen and oxygen atoms ${ }^{12-16}$ or between amino and nitrile nitrogen atoms ${ }^{17}$ in different structural units. This work, which is part of the Buruli ulcer control program, is interested in mycolactone C. It aims to determine, by the methods of Quantum Chemistry, some physicochemical properties of mycolactone C, particularly the geometric, energetic, and spectroscopic parameters of hydrogen bonding established on heteroatoms, the probable site of intermolecular interaction of the toxin. The calculations are carried out at the ONIOM level (B3LYP / 6-311 + G (d, p): AM1). The choice of Density Functional (DFT / B3LYP) gives very encouraging results. As for the selection of splitvalence and triple-zeta bases, they are sufficiently extensive. Taking into account diffuse and polarization functions is essential when it comes to intermolecular interactions. Such a study will make it possible to propose a theoretical model of annihilation of the destructive effects of mycolactone $\mathrm{C}$.

\section{Materials and Methods}

\subsection{Method and Level of Calculation}

Mycolactone $\mathrm{C}$ heteroatom numbering is done from the longest side chain, from right to left, through the lactone nucleus. Thus, the numbers 1, 2, 3, 4, 5, 6, 7, and 8 denote heteroatoms and correspond respectively to the names of the various complexes formed. Figures 1 and 2 show the $2 \mathrm{D}$ and $3 \mathrm{D}$ molecular structures of mycolactone $\mathrm{C}$, respectively.<smiles>CC=CC=CC(C)=CC=CC(=O)OC(CCCC(=O)OC(CC=C(C)CC(C)CC(C)=CCC(O)CC(C)O)[C@H](C)CC(C)CC(C)O)C(C)CC(C)O</smiles>

Figure 1. 2D structure of mycolactone C

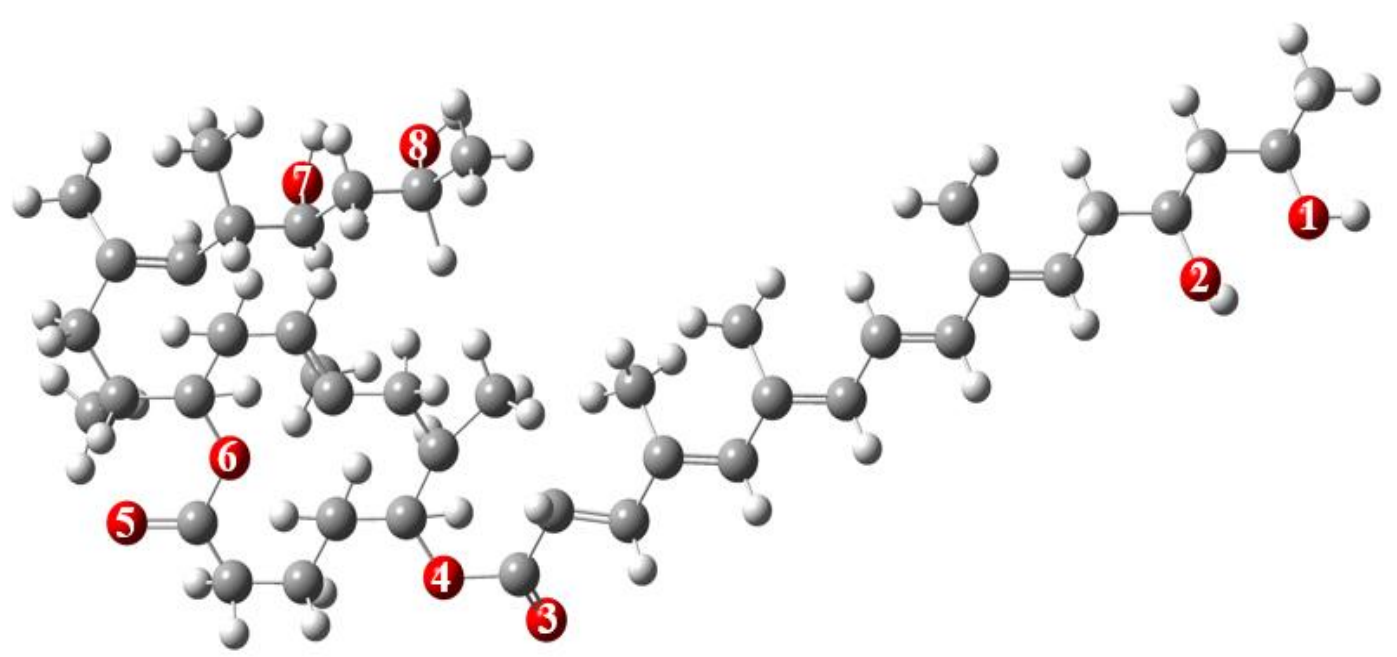

Figure 2. Molecular structure of mycolactone C visualized with Gauss-View 03 software

The ONIOM method, developed by Morokuma et al. ${ }^{18}$ is used to determine some physicochemical properties of mycolactone $\mathrm{C}$ and its various hydrogen bond complexes. The ONIOM method has often been used with success on large molecules ${ }^{18}$. It consists of dividing the studied system into several layers, each of the layers being processed at a different level of calculation. Therefore, it makes it possible to 
precisely describe the part of the system that is of particular interest for the study, so-called the internal layer or even the model system. However, it represents the rest of the system less precisely, called the external layer or environment. Figure 3 presents the description of the cutting of a mycolactone complex with the ONIOM method.

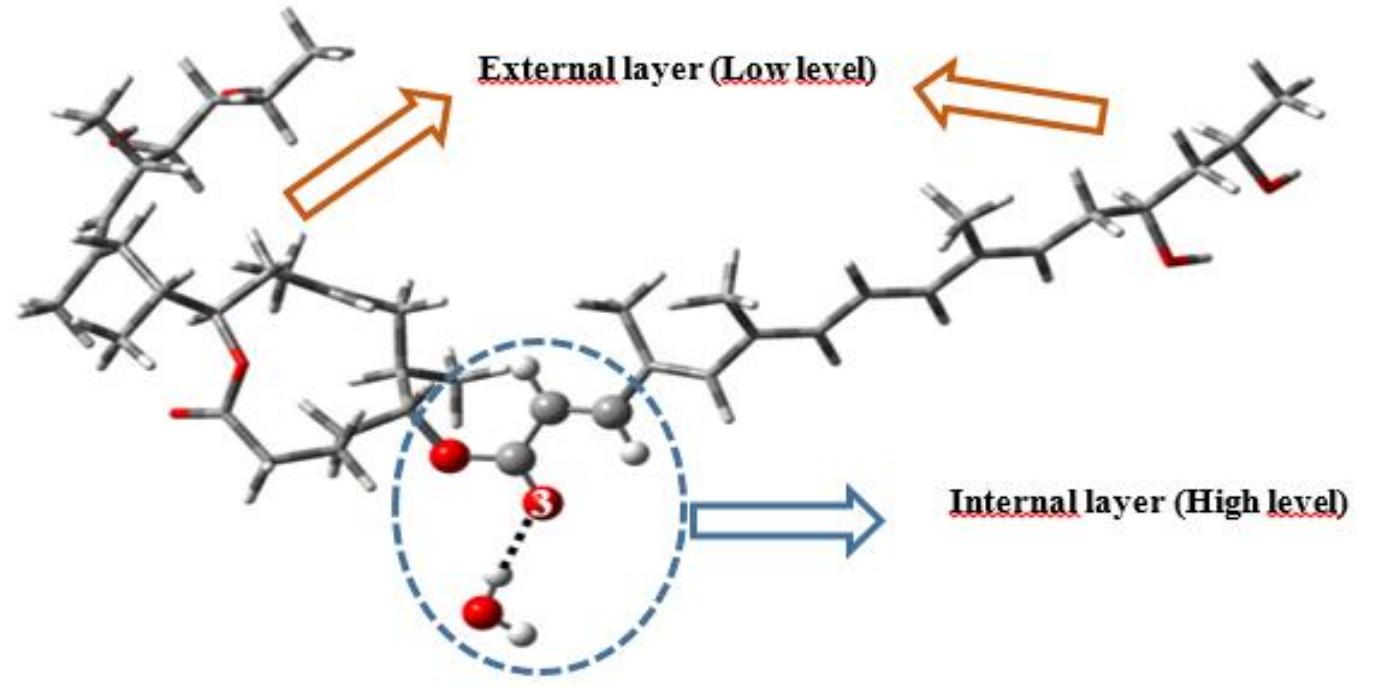

Figure 3. Model for cutting a mycolactone complex with the ONIOM 2 method

The ONIOM method makes it possible to obtain the energy of the real system at a high computational level. The total energy of the real system, determined by extrapolation, is obtained from three independent calculations. The theory of this method is based on the fact that the energy difference between a high-level calculation $\left(\boldsymbol{E}_{\text {real }}^{\text {high }}\right)$ and another low level ( $\left.\boldsymbol{E}_{\text {real }}^{\text {low }}\right)$ for the real system is equal to the energy difference between a high-level calculation $\left(\boldsymbol{E}_{\text {model }}^{\text {high }}\right)$ and a lowlevel calculation $\left(\boldsymbol{E}_{\boldsymbol{m} \text { odel }}^{\text {low }}\right.$ ) for the model system. The high and low-level calculation methods being the same in both cases, allow the following relationship to be established:

$\mathbf{E}_{\text {real }}^{\text {high }}-\mathbf{E}_{\text {real }}^{\text {low }}=\mathbf{E}_{\text {model }}^{\text {high }}-\mathbf{E}_{\text {model }}^{\text {low }}$

Knowledge of three of these terms gives access to the fourth without the need to calculate it. Therefore, we can have the energy of the real system at a high level of computation from three less expensive computations. The total interaction energy obtained from the ONIOM 2 calculation ${ }^{19}$, called extrapolated energy, is defined below:

$\mathbf{E}^{\text {ONIOM } 2} \approx \mathbf{E}_{\text {real }}^{\text {high }}=\mathbf{E}_{\text {model }}^{\text {high }}+\mathbf{E}_{\text {real }}^{\text {low }}-\mathbf{E}_{\text {model }}^{\text {low }}$

All the calculations were carried out at the ONIOM level (B3LYP / 6-311 + G (d, p): AM1) with the Gaussian 03 software ${ }^{20}$. Functional Density (DFT / B3LYP) choice makes it possible to obtain results that are relatively less heav1y but deemed adequate. The presence of diffuse and polarization functions is essential for taking into account the free doublets of heteroatoms.

\subsection{Geometry Optimization}

All the complexes of mycolactone $\mathrm{C}$ were built on each of its heteroatoms, particularly the oxygen atoms, with a water molecule as hydrogen bond donor (DLH) (Figure 4).

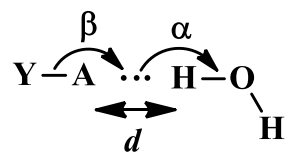

Figure 4. Geometric parameters $\mathrm{d}, \mathrm{D}, \alpha, \beta$ describing an $\mathrm{H}$ bond ${ }^{21}$

Before optimization, for all complexes, the angle of linearity $\alpha$ was set at $180^{\circ}$, and the angle of direction $\beta$ was set at $109.5^{\circ}$ for $\mathrm{sp}^{3}$ hybridized oxygen and $120^{\circ}$ for oxygen hybridized $\mathrm{sp}^{2}$, according to the Gillespie method or VSEPR method (Valence Schell Electronic Pair Repulsion) Figure 5. The distance between an oxygen atom of mycolactone $\mathrm{C}$ and a hydrogen atom of the probe $\left(\mathrm{H}_{2} \mathrm{O}\right)$ is fixed at $2 \AA$. These values correspond respectively to the angles and the minimum approach distance of the hydrogen bond ${ }^{22}$. 

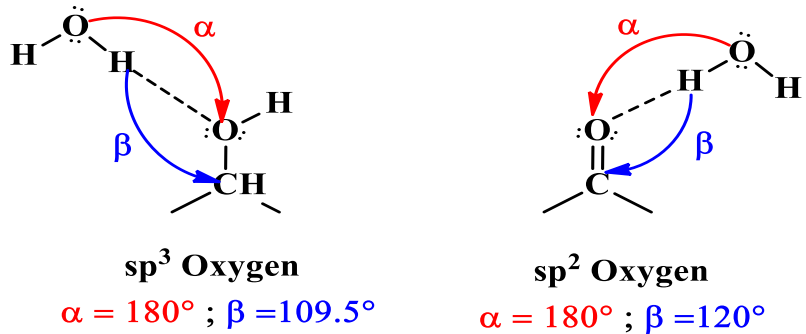

Figure 5. Definition of the angles of linearity and direction describing the interactions by the hydrogen bond on the oxygen atoms $\mathrm{sp}^{2}$ and $\mathrm{sp}^{3}$

\subsection{Energy Parameters}

Knowledge of the variations in energy contributions to internal energy at $0 \mathrm{~K}$ and $298 \mathrm{~K}$ between the product and the reactants contributes to the energetic characterization of a chemical reaction. For a given energy parameter $\mathrm{X}$, its variation is determined through the following relation:

$\Delta \mathbf{X}=\sum$ (Products) $-\sum($ Reagents $)$

The optimization of the geometries and the calculation of the frequencies of the free and interacting molecules make it possible to determine the variation of the internal energy at $0 \mathrm{~K}\left(\Delta \mathrm{E}_{0 \mathrm{~K}}\right)$ and at $298 \mathrm{~K}\left(\Delta \mathrm{E}_{298 \mathrm{~K}}\right)$ relative to the relationship studied. The variation of the internal energy at $298 \mathrm{~K}$ is a sum of the various electronic contributions of the translation, rotation, vibration, and of the internal energy at $0 \mathrm{~K}$ through the relation:

$$
\Delta \mathbf{E}_{298}=\Delta \mathbf{E}_{\text {élec }}^{\mathbf{o}}+\Delta \mathbf{E}_{\text {trans }}^{\mathbf{o}}+\Delta \mathbf{E}_{\text {rot }}^{\mathbf{o}}+\Delta \mathbf{E}_{\text {vib,thermal }}^{\mathbf{o}}+
$$$$
\Delta \mathbf{E}_{\mathbf{0 K}}
$$

Calculations of the frequencies of the optimized molecules allow access to all the components of internal energy. In addition, E0K, commonly called ZPVE (Zero Point Vibrational Energy) corresponds to the contribution of the vibration of molecules at $0 \mathrm{~K}$. It reflects the vibrational energy at the zero point induced by the normal modes of vibration of frequency vi of the $\mathrm{N}$ nuclei at $0 \mathrm{~K}$. It is defined by relation (5):

$$
\mathrm{ZPVE}=\frac{1}{2} R \sum_{\mathrm{i}=1}^{3 \mathrm{~N}-6} \frac{\mathrm{h} v_{\mathrm{i}}}{\mathrm{k}}
$$

With $3 \mathrm{~N}-6$ defined as the number of normal modes of vibration of a non-linear molecule $(3 \mathrm{~N}-5$ for a linear molecule); $\mathrm{k}$ the Boltzmann constant; $\mathrm{h}$ the Planck constant and $\mathrm{R}$ gas constant). This energy corresponds to the internal energy at $0 \mathrm{~K}\left(\Delta E_{0 K}\right)$. During a temperature rise from $0 \mathrm{~K}$ to $\mathrm{T}(\mathrm{K})$, it is necessary to consider the additional energy term corresponding to the population of vibrational energy levels during the temperature change. This term is defined by:

$\mathbf{E}_{\text {vib,therm }}^{o}=R \sum_{i=1}^{3 N-6} \frac{h v_{i} / k}{e^{\left(h v_{i} / T(K)\right)}-1}$

As for the contributions of rotation and translation, they are taken from the ideal gas approximation by this equation:

$$
\Delta \mathrm{E}_{\text {trans }}^{0}=\Delta \mathrm{E}_{\mathrm{rot}}^{0}=-\frac{3}{2} \mathbf{R T}
$$

The variation of the internal energy $\Delta \mathrm{E} 298 \mathrm{~K}$ at $298 \mathrm{~K}$ can then be written:

$$
\Delta \mathrm{E}_{298 \mathrm{~K}}^{0}=\Delta \mathrm{E}_{\text {élec }}^{0}+\Delta \mathrm{ZPVE}+\Delta \mathrm{E}_{\text {vib,thermal }}^{0}-3 \mathrm{RT}
$$

This relation deduced the enthalpy of reaction at $298 \mathrm{~K}$. It corresponds to the variation of the internal energy corrected by the term $\Delta(\mathrm{PV})$, and $\Delta \mathrm{nRT}(\Delta \mathrm{n}$ being the variation of the number of gaseous moles during the reaction).

$$
\Delta \mathbf{H}_{298 \mathrm{~K}}^{0}=\Delta \mathrm{E}_{298 \mathrm{~K}}^{\mathbf{0}}+\Delta \mathrm{nRT}
$$

The entropic contributions of $\boldsymbol{S}_{\text {trans }}$, translation, $\boldsymbol{S}_{\text {rot }}$ rotation and $\boldsymbol{S}_{\boldsymbol{v} \boldsymbol{i b}}$ vibration of a given species at $298 \mathrm{~K}$ are grouped in the total entropy term $\mathrm{S}$. The reaction entropy is determined according to the relation.

$\Delta \mathbf{S}_{298 \text { к }}=\sum S($ products $)-\sum S($ reagents $)$

However; the Gibbs energy at $298 \mathrm{~K}$, related to the reaction, is obtained by the relation below:

$\Delta G_{298 K}^{0}=\Delta H_{298 K}^{0}-T \Delta S_{298 K}^{0}$

\subsection{Spectroscopic Parameters}

Spectroscopic descriptors are generally considered to be spectroscopic scales of $\mathrm{H}$ bond basicity. Hydrogen bond causes elongation of the $\mathrm{X}-\mathrm{H}$ bond formed between the donor atom $\mathrm{X}$ and the hydrogen atom $\mathrm{H}$. These results in a decrease of a few hundred $\mathrm{cm}^{-1}$ of the wavenumber of the stretching vibration and a significant increase in intensity. However, their implementation relates to vibrational spectroscopy, where we measure the frequency shift $\Delta v(X-H)$ of the elongation vibration of the H-bond donor during the formation of the complex. When $\Delta v(X-H)$ is negative, it means that the frequency is shifting in the red for the complex, and when $\Delta v(\mathrm{X}-\mathrm{H})$ is positive, we use the term "displacement in the blue" ${ }^{23}$. The $\Delta v(O-H)$ shifts were calculated using asymmetric vibrators of the "free" water $\left(\mathrm{H}_{2} \mathrm{O}\right)$ molecule in the complex. The theoretical frequency shifts $\Delta v(O-H)$ with water were respectively determined, for the complexes formed with the oxygen atoms $\mathrm{sp}^{2}$ and $\mathrm{sp}^{3}$ by the relations below (12) and (13)

$$
\begin{aligned}
& \Delta \mathbf{v}(\mathbf{O}-\mathbf{H})=\boldsymbol{v}_{\boldsymbol{s}}(\mathbf{O}-\mathbf{H})_{\text {free }}-\mathbf{v}(\mathbf{O}- \\
& \text { H ... } \left.\boldsymbol{O}_{\text {sp2 }}\right)_{\text {complex }} \\
& \Delta \mathbf{v}(\mathbf{O}-\mathbf{H})=\boldsymbol{v}_{\boldsymbol{s}}(\mathbf{O}-\mathbf{H})_{\text {free }}-\mathbf{v}(\mathbf{O}- \\
& \text { H ... } \left.O_{\text {sp3 }}\right)_{\text {complex }}
\end{aligned}
$$


At the level of ONIOM theory (B3LYP / 6-311 + G $(\mathrm{d}, \mathrm{p})$ : AM1), the vibration frequency $v_{s}(\mathrm{O}-\mathrm{H})_{\text {free }}$ is $3923.88 \mathrm{~cm}^{-1}$

\section{Results and Discussion}

\subsection{Geometric Parameters Analysis}

The various complexes formed were optimized to determine their geometry of the equilibrium. Figure 6 illustrates the geometries of the complexes formed with the hybridized oxygen atoms $\mathrm{sp}^{2}$ or $\mathrm{sp}^{3}$ before and after the optimization.

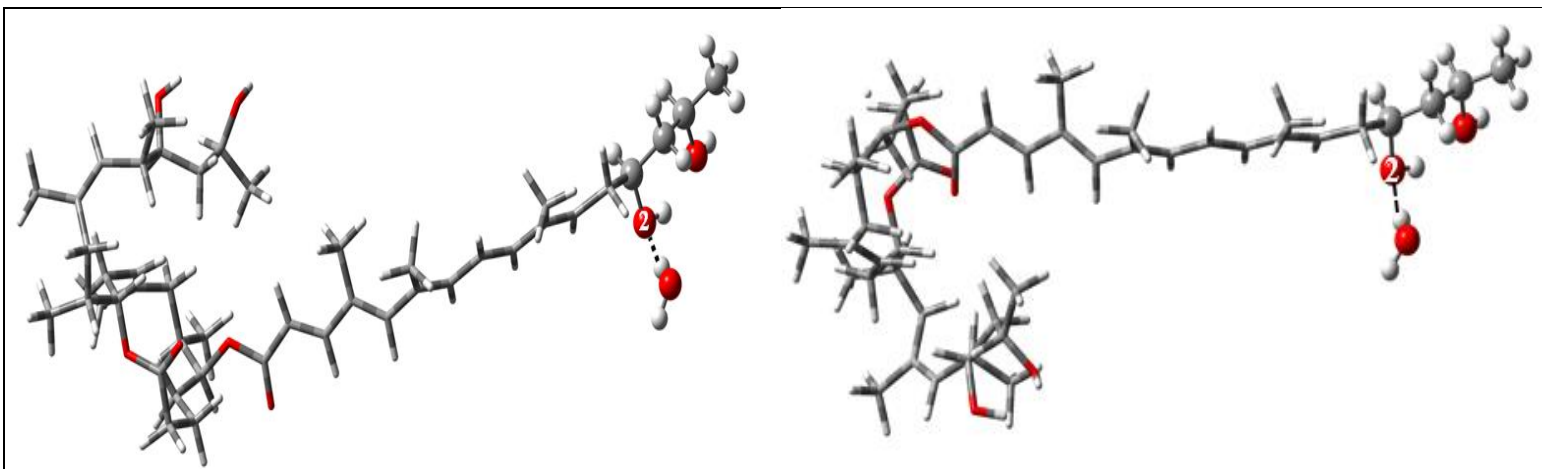

Initial (left) and optimized (right) structures of the $\mathrm{H}$ binding complex formed from mycolactone $\mathrm{C}$ on the $\mathbf{O}_{2} \mathbf{S p}^{3}$ heteroatom
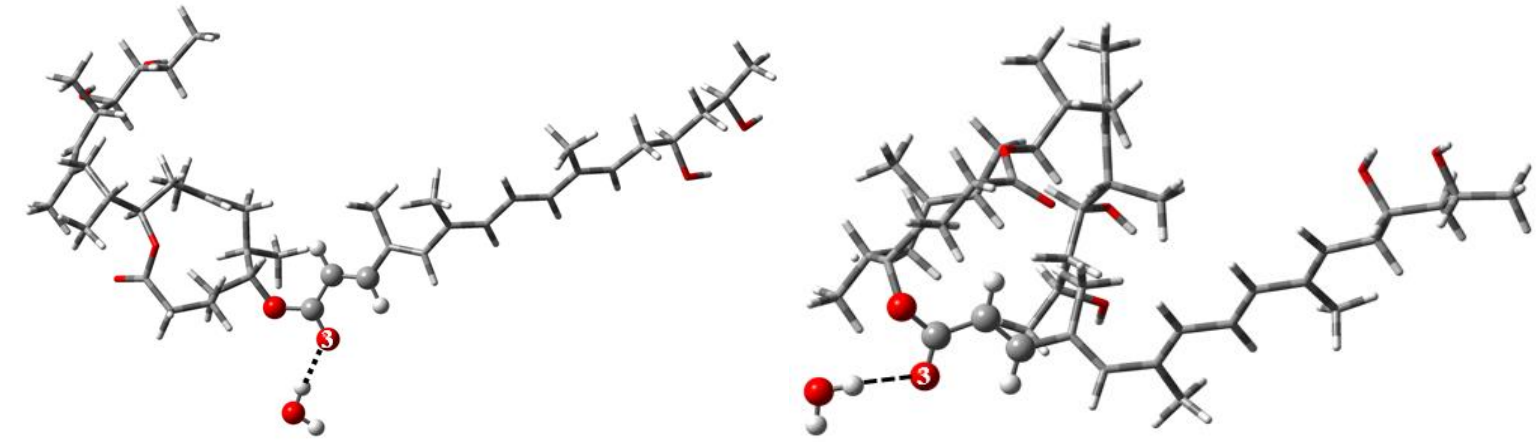

Initial (left) and optimized (right) structures of the $\mathrm{H}$ binding complex formed from mycolactone $\mathrm{C}$ on the $\mathbf{O}_{3} \mathbf{S p}^{2}$ heteroatom
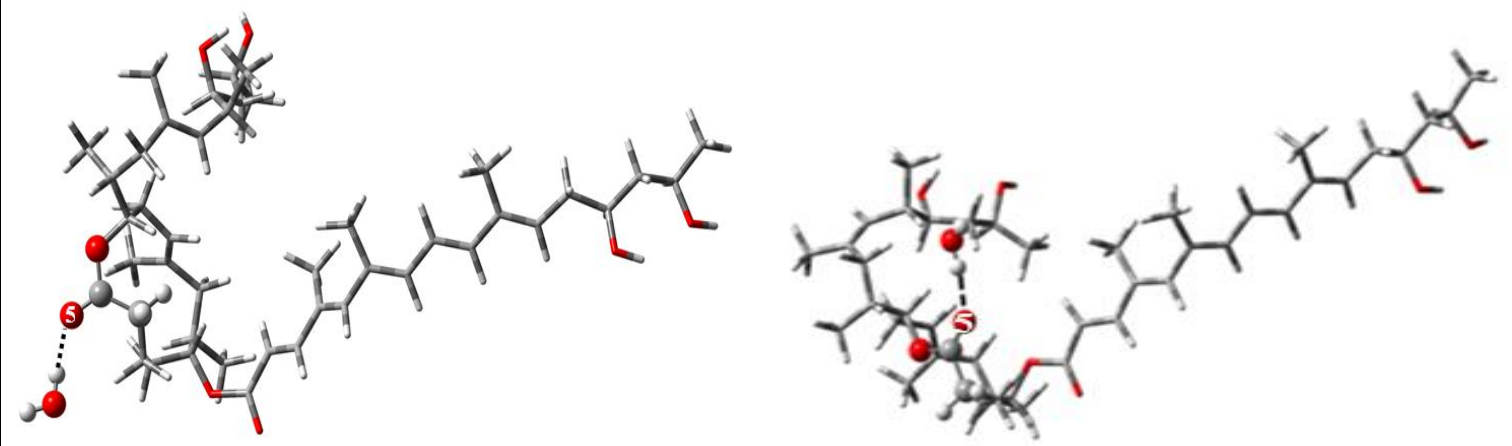

Initial (left) and optimized (right) structures of the $\mathrm{H}$ binding complex formed from mycolactone $\mathrm{C}$ on the $\mathbf{O}_{5} \mathbf{s p}^{2}$ heteroatom

Figure 6. Example of initial and optimized structures of some $\mathrm{H}$ binding complexes formed from mycolactone $\mathrm{C}$

Figure 6 was obtained from Gauss View implemented in Gaussian 03 software. The part presented in the tube is the outer layer, optimized at the low level precisely in AM1. The part presented in "ball and stick" is the model system. It is optimized at the B3LYP / 6-311 + G (d, p) level.

The visualization of the different optimized geometries of the $\mathrm{H}$ binding complexes (Figure 6) made it possible to observe that the optimizations of the geometries of the complexes were successful for all the heteroatoms of mycolactone $\mathrm{C}$. This phenomenon can be explained by the lack of mesomerism around the heteroatoms. This lack of mesomerism around these heteroatoms reinforces their basicity. 
Table 1. Geometric parameters of mycolactone $\mathrm{C}$ hydrogen bond complexes calculated at the ONIOM level (B3LYP / 6-311 + G (d, p): AM1).

\begin{tabular}{|c|c|c|c|}
\hline & \multicolumn{3}{|c|}{ MYCOLACTONE C } \\
\hline & $\alpha\left(e n^{\circ}\right)$ & $\beta\left(e^{\circ}\right)$ & $d(e n \AA)$ \\
\hline $\mathbf{O}_{1} \mathbf{s p}^{3}$ & 140.60 & 114.80 & 2.52 \\
\hline $\mathrm{O}_{2} \mathbf{S p}^{3}$ & 167.00 & 110.30 & 1.90 \\
\hline $\mathrm{O}_{3} \mathrm{sp}^{2}$ & 170.40 & 125.25 & 1.98 \\
\hline $\mathrm{O}_{4} \mathbf{S p}^{3}$ & 135.90 & 137.80 & 2.59 \\
\hline $\mathrm{O}_{5} \mathrm{Sp}^{2}$ & 168.90 & 118.80 & $\underline{1.87}$ \\
\hline $\mathrm{O}_{6} \mathrm{Sp}^{3}$ & 131.20 & 156.51 & 2.84 \\
\hline $\mathbf{O}_{7} \mathbf{S p}^{3}$ & 126.90 & 126.29 & 2.28 \\
\hline Ossp ${ }^{3}$ & 170.50 & 117.90 & 1.99 \\
\hline
\end{tabular}

Table 1 shows that, the heteroatoms $\mathbf{O}_{2} \mathbf{S p}^{\mathbf{3}}$ $\left(\alpha=167.00^{\circ} ; \beta=110.30^{\circ}\right), \mathbf{O}_{3} \mathbf{S p}^{2}\left(\alpha=170.40^{\circ}\right.$; $\left.\beta=125.25^{\circ}\right), \mathbf{O}_{5 \mathbf{s p}} \mathbf{2}^{(}\left(\alpha=168.90^{\circ}, \beta=118.80^{\circ}\right)$ and $\mathbf{O s s p}^{3}\left(\alpha=170.50^{\circ} ; \beta=117.90^{\circ}\right)$, exhibiting high values of linearity angles of and direction are likely to create strong hydrogen bonds. On the other hand, the heteroatoms $\mathbf{O}_{1} \mathbf{S p}^{\mathbf{3}}\left(\alpha=140.60^{\circ} ; \beta=114.80^{\circ}\right), \mathbf{O}_{4} \mathbf{S p}^{\mathbf{3}}$ $\left(\alpha=135.90^{\circ} ; \beta=137.80^{\circ}\right), \mathbf{O}_{6 \mathbf{S p}}{ }^{3}\left(\alpha=131.20^{\circ}\right.$; $\left.\beta=156.51^{\circ}\right)$ and $\mathbf{O}_{7} \mathbf{S p}^{3}\left(\alpha=126.90^{\circ} ; \beta=126.29^{\circ}\right)$ are heteroatoms that do not make strong hydrogen bonds because of the low and far angle values of linearity and direction standard values.

Regarding the lengths of the $\mathrm{H}$ bonds (distance $\mathrm{d}$ ), the practice is to consider a contact as an $\mathrm{H}$ bond if the distance $d$ is less than the sum of the Van der Waals radii, taking respectively $1.52 \AA$, for oxygen atoms and $1.0 \AA$ for hydrogen atoms; or $\mathrm{d} \leq 2.52 \AA$ for contact with oxygen ${ }^{24}$. It is also known that the $\mathrm{H}$ bond is as strong as its distance is short. The heteroatoms $\mathbf{O}_{\mathbf{1}} \mathbf{S p}^{\mathbf{3}}$ $(2.52 \AA), \mathbf{O}_{2} \mathbf{s p}^{3}(1.90 \AA), \mathbf{O}_{3} \mathbf{s p}^{2}(1.98 \AA), \mathbf{O}_{5} \mathbf{s p}^{2}$ $(1.87 \AA), \mathbf{O}_{7} \mathbf{S p}^{3}(2.28 \AA)$, and $\mathbf{O}_{8} \mathbf{s p}^{3}$ (1.99 $\AA$ ) having some hydrogen bonds length less than the sum of the Van der Waals radii, are likely to be some sites of strong hydrogen bonding. Only the $\mathbf{O}_{4} \mathbf{S p}^{3}$ (2.59 $\AA$ ) and $\mathbf{O}_{6} \mathbf{S p}^{\mathbf{3}}(2.84 \AA)$ heteroatoms, having distance values $d$ greater than the sum of the Van der Waals radii, are not probable sites for the creation of a strong hydrogen bond. Therefore, the $\mathbf{O}_{5} \mathbf{s p} \mathbf{p}^{2}$ heteroatom $(\mathbf{1 . 8 7} \AA)$, with the smallest d distance value, creates a perfect affinity with the hydrogen of the probe.

The analysis of the geometric parameters indicates that the heteroatom $\mathbf{O}_{5} \mathbf{S p}^{\mathbf{2}}$ is the most probable site of creating a strong hydrogen bond because of the lower values of distance $d$ and the good values of the angles of linearity and the direction observed.

\subsection{Analysis of Energy Parameters}

The calculations carried out based on functions on the probe (water molecule), and the $\mathrm{H}$ binding complexes were carried out with the Gaussian 03 software in a vacuum with the ONIOM method at the ONIOM level (B3LYP / 6-311 + G (d, p): AM1). The molecular structure of each of the obtained $\mathrm{H}$ binding complexes has been optimized. A frequency calculation follows this step. The validation of the bases of functions and the levels of calculation chosen necessarily passes by calculating the error. The error (Err) estimate in the ONIOM method is defined as the difference in energy between the real and model systems. It looks like this:

$\mathbf{E}_{\mathrm{rr}}=\mathbf{E}^{\text {ONIOM 2 }}-\mathbf{E}_{\text {réel }}^{\text {haut }}$

The more the error is equal to zero or close to zero, the more the ONIOM method gives excellent approximations. The base chosen for the low level combines perfectly with that chosen for the high level. However, suppose the error is different from zero or very far from zero. In that case, the selected bases for the two levels are not compatible, and the ONIOM method cannot produce good approximations. Thus, the ONIOM method used at these levels and these bases converge towards the expected results. Therefore, after several tests of calculations carried out with different bases and different levels on different systems, we calibrated the study of the other intermolecular descriptors of mycolactone $\mathrm{C}$ at the level (B3LYP / 6-311 + G (d, p): AM1). Because for this level, the calculated values of the error are close to zero $(\mathrm{E}$ rr $\approx 0)$. Better still, the values of the electronic energies (EоNIOM2) obtained were subsequently used to calculate the thermodynamic interaction quantities of mycolactone $\mathrm{C}$. These results testify to the good use of the ONIOM method described by Keiji Morokuma et al.

The energy parameters determined are the variations of the thermodynamic quantities linked to the complexation by the $\mathrm{H}$ bond of the sites considered. 
Table 2. Energy parameters of H-bond complexation of different heteroatoms. Quantities (in $\mathrm{kJ} / \mathrm{mol}$ ), Complexation entropy values were expressed in $((\mathrm{kJ}) /(\mathrm{mol} . \mathrm{K}))$.

\begin{tabular}{|c|c|c|c|}
\hline & \multicolumn{3}{|c|}{ MYCOLACTONE C } \\
\hline & $\Delta_{\mathbf{r}} \mathbf{H}_{298}^{\circ}$ & $\Delta_{r} S_{298}^{\circ}$ & $\Delta_{\mathbf{r}} \mathbf{G}_{298}^{\circ}$ \\
\hline $\mathbf{O}_{1} \mathbf{s p}^{3}$ & -15.57 & -0.12 & 20.22 \\
\hline $\mathbf{O}_{2} \mathbf{s p}^{3}$ & -29.94 & -0.06 & -12.06 \\
\hline $\mathrm{O}_{3} \mathrm{sp}^{2}$ & -35.96 & -0.07 & -15.10 \\
\hline $\mathrm{O}_{4} \mathrm{sp}^{3}$ & -02.77 & -0.09 & 24.05 \\
\hline $\mathrm{O}_{5} \mathrm{sp}^{2}$ & $\underline{-44.28}$ & -0.09 & $\underline{-17.46}$ \\
\hline $\mathrm{O}_{6 \mathrm{Sp}} \mathrm{p}^{3}$ & 19.32 & -0.14 & 61.04 \\
\hline $\mathbf{O}_{7} \mathbf{s p}^{3}$ & -30.53 & -0.06 & -12.65 \\
\hline Ossp ${ }^{3}$ & -15.75 & -0.12 & 20.01 \\
\hline
\end{tabular}

The exothermicity of each of the complexation reactions of the water molecule on all the different sites of mycolactone $\mathrm{C}$ is reflected by the negative values of the complexation enthalpies. All heteroatoms except $\mathrm{O}_{6} \mathrm{sp}^{3}(19.32 \mathrm{~kJ} / \mathrm{mol})$ show negative enthalpy of complexation values. This indicates that the complexation reactions with these sites are exothermic. On the other hand, on the $\mathrm{O}_{6} \mathrm{sp}^{3}$ heteroatom $(19.32 \mathrm{~kJ} / \mathrm{mol})$ the complexation reaction is not exothermic depending on the positive value of the enthalpy of complexation.

Regarding the free enthalpy of complexation, only the heteroatoms $\quad \mathrm{O}_{2} \mathrm{sp}^{3} \quad(-12.06 \mathrm{~kJ} / \mathrm{mol}), \quad \mathrm{O}_{3} \mathrm{sp}^{2}$ $(-15.10 \mathrm{~kJ} / \mathrm{mol}), \mathrm{O}_{5} \mathrm{sp}^{2}(-17.46 \mathrm{~kJ} / \mathrm{mol})$, and $\mathrm{O}_{7} \mathrm{sp}^{3}$ $(-12,65 \mathrm{~kJ} / \mathrm{mol})$ show negative values. These values indicate that the reactions evolve in the direction of the spontaneous formation of the complexes at a temperature of $298.15 \mathrm{~K}$ and a pressure of $1 \mathrm{~atm}$. It should be noted that the lowest value of free enthalpy of complexation is observed with the heteroatom $\mathrm{O}_{5} \mathrm{sp}^{2}(-\mathbf{1 7 . 4 6} \mathbf{~ k J} / \mathbf{m o l})$. In contrast, the heteroatoms $\mathrm{O}_{1} \mathrm{sp}^{3}(20.22 \mathrm{~kJ} / \mathrm{mol}), \mathrm{O}_{4} \mathrm{sp}^{3}(24.05 \mathrm{~kJ} / \mathrm{mol}), \mathrm{O}_{6} \mathrm{sp}^{3}$ $(61.04 \mathrm{~kJ} / \mathrm{mol})$, and $\mathrm{O}_{8} \mathrm{sp}^{3}(20.01 \mathrm{~kJ} / \mathrm{mol})$, showing values positive enthalpies free from complexation, are not favorable sites for spontaneous reactions with the electrophilic site of the probe. For a comparative analysis, we note that the two heteroatoms $\mathrm{O}_{1} \mathrm{sp}^{3}$ and $\mathrm{O}_{8} \mathrm{sp}^{3}$ located at the ends of the two side chains present positive values of free enthalpy of complexation. This means that these two heteroatoms, which are derived from hydroxyl groups, cannot set up reactions in the direction of the formation of complexes. This information is also verified through their hydrogen bond length values, which appear to be highest for the four heteroatoms derived from hydroxyl groups. Concerning heteroatoms $\mathrm{O}_{2} \mathrm{sp}^{3}$ and $\mathrm{O}_{7} \mathrm{sp}^{3}$, they exhibit negative values of free enthalpy of complexation, therefore favorable to creating strong hydrogen bonds. The difference in the free enthalpy of complexation values observed for the $\mathrm{O}_{1} \mathrm{sp}^{3}$ and
$\mathrm{O}_{2} \mathrm{sp}^{3}$ heteroatoms lies in the difference in the values of their net charge and the phenomenon of steric hindrance. The oxygen atoms $\mathrm{O}_{7} \mathrm{sp}^{3}$ and $\mathrm{O}_{8} \mathrm{sp}^{3}$, being in the same spatial arrangements as the atoms $\mathrm{O}_{1} \mathrm{sp}^{3}$ and $\mathrm{O}_{2} \mathrm{sp}^{3}$, benefit from the same explanations because they are also located at the end of the second side chain.

We also find that the heteroatoms $\mathrm{O}_{4} \mathrm{sp}^{3}$ and $\mathrm{O}_{6} \mathrm{sp}^{3}$ exhibit positive values of free enthalpy of complexation. They are therefore not favorable to the creation of a hydrogen bond. This is also verified through their hydrogen bond length values, which remain the highest of all heteroatoms' hydrogen bond length values. $\mathrm{sp}^{2}$ hybridized heteroatoms $\left(\mathrm{O}_{3} \mathrm{sp}^{2}\right.$, $\mathrm{O}_{5} \mathrm{sp}^{2}$ ) exhibit negative complexation enthalpy values. This means that there is a possibility of a spontaneous reaction between these heteroatoms and the electrophilic site of the probe. But the lowest value of free enthalpy of complexation of these two heteroatoms is observed with the $\mathrm{O}_{5} \mathrm{sp}^{2}$ heteroatom. This shows that the $\mathrm{O}_{5} \mathrm{sp}^{2}$ heteroatom located in the nucleus or chromophore of mycolactone $\mathrm{C}$ is the most nucleophilic site. It easily sheds one of its doublets and reacts quickly with the acid in the probe, helping to create a strong hydrogen bond.

After examining the values of the energy complexation parameters, the heteroatom $\mathrm{O}_{5} \mathrm{sp}^{2}$ having the lowest value of the free enthalpy of complexation $(-17.46 \mathrm{~kJ} / \mathrm{mol}$, gives the most stable complex.

\subsection{Analysis of Spectroscopic Parameter}

Table 3 shows that the vibration frequency $v_{s}(\mathrm{O}-\mathrm{H})_{\text {free }}$ calculated at the ONIOM level (B3LYP / 6-311 + G (d, p): AM1) is $3923.88 \mathrm{~cm}^{-1}$.

The strongest value of the variations in vibration frequencies is observed on the $\mathbf{O}_{5} \mathbf{S p}^{2}$ heteroatom $\left(223.10 \mathrm{~cm}^{-1}\right)$. 
Table 3. Variations of the theoretical frequencies $\Delta v(\mathrm{O}-\mathrm{H})\left(\mathrm{cm}^{-1}\right)$ calculated at the level of theory ONIOM (B3LYP / 6-311 + G (d, p): AM1) on mycolactone C.

\begin{tabular}{|l|l|}
\hline \multicolumn{2}{|c|}{ Mycolactone C } \\
\hline $\mathbf{O}_{\mathbf{1}} \mathbf{s p}^{\mathbf{3}}$ & 212.54 \\
\hline $\mathbf{O}_{\mathbf{2}} \mathbf{s p}^{\mathbf{3}}$ & 212.64 \\
\hline $\mathbf{O}_{\mathbf{3}} \mathbf{s p}^{\mathbf{2}}$ & 222.47 \\
\hline $\mathbf{O}_{\mathbf{4} \mathbf{s}^{\mathbf{3}}}$ & 191.05 \\
\hline $\mathbf{O}_{\mathbf{s}} \mathbf{s}^{\mathbf{2}}$ & $\underline{\mathbf{2 2 3 . 1 0}}$ \\
\hline $\mathbf{O}_{\mathbf{6}} \mathbf{s p}^{\mathbf{3}}$ & 222.02 \\
\hline $\mathbf{O}_{7} \mathbf{s p}^{\mathbf{3}}$ & 218.25 \\
\hline $\mathbf{O}_{\mathbf{s}}$ & 198.93 \\
\hline
\end{tabular}

This behavior reflects the attraction effect exerted by the acceptor atom $\left(\mathrm{O}_{5} \mathrm{sp}^{2}\right)$ of the monomer on the hydrogen of the probe. Moreover, it has been established that the greater the variation in vibration frequencies, the stronger the $\mathrm{H}$ bond. This observation shows that the $\mathbf{O}_{\mathbf{5}} \mathbf{s} \mathbf{p}^{2}$ heteroatom is the preferred site for a stronger $\mathrm{H}$ binding.

The analysis of the values of the geometric, energetic, and spectroscopic parameters clearly shows that the $\mathbf{O}_{\mathbf{5}} \mathbf{s p}^{2}$ heteroatom is the best site of interaction by hydrogen bonding of this toxin.

The reliability of our level of computation is in harmony with the work carried out by Maeda et al. ${ }^{25}$. With them, the ONIOM method was implemented to determine the energies of dissociation of bonds of organic molecules or the chemical reactivity of carbon nanotubes. The validity of this method has also been demonstrated for purely organic compounds by comparing the theoretical results with those obtained using a more sophisticated level of theory and experimental data.

\section{Conclusion}

The values of the intermolecular descriptors were made possible thanks to the ONIOM method. The first part of this study was about studying all the geometric and energetic descriptors of mycolactone $\mathrm{C}$ at the ONIOM level (B3LYP / 6-311 + G (d, p): AM1). We have highlighted through our findings that oxygen atom $\mathrm{O}_{\mathbf{5}} \mathbf{S p}^{2}$ is the probable center of the interaction of mycolactone $\mathrm{C}$. The analysis of the spectroscopic parameters of the hydrogen bond complexes, such as the shifts of the vibration frequency $\Delta v(O-H)$ with water in the second part of our study, has also confirmed that the $\mathbf{O}_{\mathbf{5}} \mathbf{S \mathbf { p } ^ { 2 }}$ heteroatom is the site that establishes the stronger attraction effect with the hydrogen of the probe. Based on these results, the $\mathbf{O}_{\mathbf{5}} \mathbf{S p}^{\mathbf{2}}$ heteroatom was identified as the suitable and likely site of intermolecular interaction. These researches are real baselines and promising for researchers to overcome the destructive of mycolactones. Future investigations will be interesting to undertake some research that will mainly focus on further understanding the interaction of mycolactone $\mathrm{C}$ through the oxygen atom $\mathbf{O}_{\mathbf{5}} \mathbf{S p}^{\mathbf{2}}$; for better treatment of Buruli ulcer. Moreover, it is now possible to improve these results on the theoretical characterization of the physicochemical properties of mycolactone $\mathrm{C}$ by using other levels of calculation with more extensive bases. Thus, we are planning shortly;

- to extend the study of hydrogen bonding interactions to other mycolactones to better understand their inhibitory effects as well as their biological activities;

- to characterize the protonation interactions of different mycolactones complexes to predict their stays in the fats of the subcutaneous tissues.

\section{References}

1- G. E. Anagonou, G. E. Sopoh, C. A. Biaou, Y. T. Barogui, A. C. Wadagni, S. R. Gnimavo, G. A. Ayelo, K. E. Saka, J. G. Houezo, R. C. Johnson, Environmental and behavioural factors associated with Mycobacterium ulcerans infection in the district of Lalo in Benin: A case-control study, Journal of Public Health and Epidemiology, 2021, 13, 54-63.

2- H. Simpson, K. Deribe, N. T. Earnest, P. Adebayo, M. Issaka, M. Frimpong, R. Ampadu, R. Phillips, P. Sauderson, J. Carno, Mapping the global distribution of Buruli ulcer : a systematic review with evidence concensus, The Lancet Global Health, 2019, 7, 912-922.

3- M. Beissner, K. L. Huber, K. Badziklou, W. A. Halatoko, I. Maman, F. Vogel, E. Piten, K. Helfrich, C. Mengele, J. Nitschke, K. Amekuse, F. X. Wiedemann, A. Dietfenhardt, B. Kobara, K. H. Herbinger, A. B. Kere, M. Prince-David, G. Bretzel, Loop-Mediated Isothermal Amplification for Laboratory Confirmation of Buruli Ulcer Disease-Towards a Point-of-Care Test, PLoS Negl Trop Dis., 2015, 9, 4219-4230.

4- M. Beissner, K. L. Huber, K. Badziklou, W. A. Halatoko, I. Maman, F. Vogel, E. Piten, 
K. Helfrich, C. Mengele, J. Nitschke, K. Amekuse, F. X. Wiedemann, A. Dietfenhardt, B. Kobara, K. H. Herbinger, A. B. Kere, M. Prince-David, G. Bretzel, Implementation of a national reference laboratory for Buruli ulcer disease in Togo, PLoS Negl Trop Dis., 2013, 7, 1371-1380.

5- M. A. Kabiru, S. Tjip, R. O. van der Werf, F. S. Phillips, J. Sarfo, S. O. Abotsi, W. N. Mireku, K. Asiedu, Y. Stienstra, K. Sandor-Adrian, Short Report: Buruli Ulcer Control in a Highly Endemic District in Ghana: Role of CommunityBased Surveillance Volunteers, Am. J. Trop. Med. Hyg., 2015, 92, 115-117.

6- B. Forester, G. Demangel, T. Thye, Mycolactone induces cell death by SETD1B dependent degradation of glutathone, PLoS Negl Trop Dis., 2020, 14, 87-99.

7- A. Sami, B. Neji, J. Bassem, Derivatives of indoles in anti-tumor activities, Mar. J. Heterocycl Chim., 2020, 19, 124-133.

8- H. Marzieh, A. T. Avat, Structural Assessment of Hydrogen Bonds on Methylpentynol-Azide Clusters To Achieve Regiochemical outcome of 1,3-Dipolar cycloaddition Reactions Using Density Functional, ACS Publications, American Chemical Society, 2020, 5, 5964-5975.

9- G. R. Desiraju, Designer Crystal: intermolecular interactions, network structures and supramolecular synthons, chemical Communications, 1997, 16, 1475-1482.

10-B. Moulton, M. J. Zaworotko, From Molecules to crystal Engineering: Supramolecular Isomerism and Polymorphism in Network Solids, Chem. Rev., 2001, 101, 1629-1658.

11-I. V. Ananyev, N. A. Bokach, V. Y. Kukushkin, Structure-directing sulfur...Metal noncovalent semicoordination bonding, Acta. Cryst., 2020, 76, 436-449.

12-A. E. Bela, E. M. J. Pereira, C. T. P. Freire, N. D. Argyriou, J. Eckert, Hydrogen bonds in crystalline D-alanine: diffraction and spectroscopic evidence for difference between enantiomers, Chemistry Crysteng, 2020, 5, 6-12.

13-M. G. R. Koné, S. T. Affi, N. Ziao, K. Bamba, E. F. Assanvo, Hydrogen bonding sites in Benzimidazolyl-chalcones molecules: An ab initio and DFT investigation, Journal of Chemical and Pharmaceutical Research, 2015, 7, 805-812.

14-S. T. Affi, N. Ziao, K. Bamba, Détermination, par des méthodes ab initio et dft, des sites et énergies de protonation d'une série de molécules d'imidazopyridinyl-chalcones substituées, European Scientific Journal, 2015, 11, 138-148.

15-I. Nobeli, S. L. Price, J. P. M. Lommerse, R. Taylor, Hydrogen bonding properties of oxygen and nitrogen acceptors in aromatic heterocycles, J. Comput. Chem., 1997, 18, 20602074.
16-N. Ziao, C. Laurence, J. Y. Le Questel, Amino nitrogen and carbonyl oxygen in competitive situations, Cryst. Eng. Comm., 2002, 4, 326-335.

17-N. Ziao, J. Graton, C. Laurence, J. Y. Le Questel, Amino and Cyano $\mathrm{N}$ atoms in competitive situations, Acta Cryst., 2001, 57, 850-858.

18-K. Morokuma, ONIOM and Its Applications to Material Chemistry and Catalyses, Bulletin of the Korean Chemical Society, 2003, 24, 797-801.

19-L. W. Chung, H. Hirao, X. Li, K. Morokuma, The ONIOM method: its foundation and applications to metalloenzymes and photobiology, Computational Molecular Science, 2012, 2, 327-350.

20-M. J. Frisch, G. W. Trucks, H. B. Schlegel, G. E. Scuseria, M. A. Robb, J. R. Cheeseman, J. A. Montgomery, T. Jr., Vreven, K. N. Kudin, J. C. Burant, J. M, Millam, S. S. Iyengar, J. Tomasi, V. Barone, B. Mennucci, M. Cossi, G. Scalmani, N. Rega, G. A. Petersson, H. Nakatsuji, M. Hada, M. Ehara, K. Toyota, R. Fukuda, J. Hasegawa, M. Ishida, T. Nakajima, Y. Honda, O. Kitao, H. Nakai, M. Klene, X. Li, J. E. Knox, H. P. Hratchian, J. P. Cross, C. Adamo, J. Jaramillo, R. Gomperts, R. E. Stratmann, O. Yazyev, A. J. Austin, R. Cammi, C. Pomelli, J. W. Ochterski, P. Y. Ayala, K. Morokuma, G. A. Voth, P. Salvador, J. J. Dannenberg, V. G. Zakrzewski, S. Dapprich, A. D. Daniels, M. C. Strain, O. Farkas, D. K. Malick, A. D. Rabuck, K. Raghavachari, J. B. Foresman, J. V. Ortiz., Q. Cui, A. G. Baboul, S. Clifford, J. Cioslowski, B. B. Stefanov, G. Liu, A. Liashenko, P. Piskorz, I. Komaromi, R. L. D. J. Martin, Fox, T. Keith, M. A. Al-Laham, C. Y. Peng, A. Nanayakkara, M. Challacombe, P. M. W. Gill, B. Johnson, W. Chen, M. W. Wong, C. Gonzalez, J. A. Pople, Gaussian 03, Revision B.02. Gaussian, Inc., Pittsburgh PA, 2003.

21-W. Piao, S. Tsuda, Y. Tanaka, S. Maeda, F. Liu, S. Takahashi, T. Komatsu, Development of azobased fluorescent probes to detect different levels of hypoxia, Angewandte Chemie International Edition, 2013, 52, 13028-13032.

22-J. Sanchez-Marquez, New advances in conceptual-DFT: an alternative way to calculate the Fukui function and dual descriptor, The Journal of Molecular Modeling, 2019, 25, 19-40.

23-S.Tothadi, G. R. Desiraju, Designing ternary cocrystals with hydrogen bonds and halogen bonds, Chemical Communications, 2013, 49, 7791-7793.

24-R. Fujiyama, A. Inoule, M. Harada, Transmission Mechanism of Electronic Effects in Cyclophenylene Framework, Journal of Computer Chemistry, 2019, 18, 147-149.

25-S. Maeda, Y. Harabuchi, Y. Ono, T. Taketsugu, K. Morokuma, Intrinsic reaction coordinate: calculation, bifurcation, and automated search, International Journal of Quantum Chemistry, 2015, 115, 258-269. 